\title{
Virtual University: A Peer to Peer Open Education Network
}

\author{
Amir r. Razavi \\ Department of Computing, School of Electronics and Physical \\ Sciences, University of Surrey, Guildford, Surrey, UK
}

a.razavi, p.krause@surrey.ac.uk

\author{
Abbas Strømmen-Bakhtiar \\ Centre for Enterprise Architecture \& Information Systems, \\ Bodø Graduate School of Business, \\ University of Nordland, Bodø, Norway
}

Abbas.Strommen-Bakhtiar@uin.no

\section{Paul Krause \\ Department of Computing, School of Electronics and Physical Sciences, University of Surrey, Guildford, Surrey, UK \\ p.krause@surrey.ac.uk \\ Executive Summary}

The world is currently going through a transitional period, moving from the Service era to the Information era. Rapid societal and technological innovations are changing the way we live, communicate, and work. As the rate of the technological/societal change increases, pressure on educational institutions also increases. This pressure is fundamentally about time-lag: the time it takes for educational institutions to accumulate the necessary information, create the necessary knowledge, and then transmit it to the students.

To address this problem, we propose the concept of the Virtual University. In such a model, different universities can collaborate in an interconnected environment (Open Education Network), to present their services to one another and the public. They can use each other's educational services to create new courses, new qualifications, and new integrated (virtual) universities. Open Education Network can eliminate geographical limitations and boundaries and can offer integrations of different courses when each of them may be presented by different educational institu-

Material published as part of this publication, either on-line or in print, is copyrighted by the Informing Science Institute. Permission to make digital or paper copy of part or all of these works for personal or classroom use is granted without fee provided that the copies are not made or distributed for profit or commercial advantage AND that copies 1) bear this notice in full and 2) give the full citation on the first page. It is permissible to abstract these works so long as credit is given. To copy in all other cases or to republish or to post on a server or to redistribute to lists requires specific permission and payment of a fee. Contact Publisher@InformingScience.org to request redistribution permission.

\section{tions (customization). Degrees could} potentially be offered through the composition of various courses offered by different universities or other educational institutions. In addition, a Virtual University would be able to offer continuing education to knowledge workers in a more convenient manner. Businesses, especially innovation driven Small and Medium Enterprises (SMEs), will be another beneficiary of this sys- 
tem. SMEs can educate their staff according to their needs. They can choose individual courses or a combination of courses that address their knowledge needs, without requiring their staff to go through normal university channels. Special degrees could be constructed to acknowledge these special educational requirements.

Another benefit that accrues from this system is the lower cost of delivering educational services, since the production cost of the first course will almost constitute the total cost of the product for the complete time duration during which that product is offered (digital products which can be reused and rebroadcast and to which extensions to the contents can be easily added). There is of course the additional cost of keeping the course updated, but this is inherently less than the existing system. There is also the possibility to offer professional up-to-date educational services by the creators of the new technologies. For example, such products as computer programming, data communication, certification courses, and a host of other products can be delivered by the very people who are creating these new systems and/or products. In other words, this system (Virtual University) will reduce the time lag that exists between knowledge generation and transmission.

The stability of this network is one of the most important factors in evaluating the viability of a Virtual University. Hence, we have designed the necessary components, offered the interaction model, and focused on optimized, stable network ontology. This project is the expected expansion of knowledge-based system in the digital ecosystems' projects DBE

(http://www.surrey.ac.uk/computing/research/projects/DBE.htm) and OPAALS (http://www.surrey.ac.uk/computing/research/projects/opaals.htm). The actual implementation has been introduced as the "OKS" in the OPAALS project. (The OKS implementation can be accessed at http://www.opaals-oks.eu/). The Peer to Peer interaction model of the OPAALS project has offered us the fully distributed model to compose different services from a variety of service providers. In the Virtual University we try to extend a similar facility for educational institutions; we have considered the potential complexity on the network. In this paper, we have tried to apply inspiration to the OPAALS model and, at the same time, to overcome the network challenges.

Keywords: Virtual University, P2P network, Open Education Network, distributed conceptual models, VPIN, Digital Ecosystem, Social Network Services, Open Knowledge System.

\section{Introduction}

In 1949, Jeann Fourastié (1907-1990), the renowned French economist, presented an interesting view of the $21^{\text {st }}$ century society in which he predicted that, according to the "laws of production and consumption", the service industry would rise to become the dominant sector in the economy. The developments in the past 50 years have to a large degree proven his hypothesis to be right. During this period, we have witnessed (in the developed economies) the transition from secondary (industrial) sector to the service sector.

Each transition, be it from primary (agriculture, raw material extraction) to secondary (manufacturing) or from secondary to tertiary (services), requires a tremendous readjustment in a society. Whole new industries are created that depend on new machinery or processes, all of which demand new skilled workers. The old skills suddenly lose their value, and new skills become invaluable. This change in demand puts pressure on educational institutions to provide the education that enables the labour force to fill the newly created positions in the labour market.

For educational institutions to be effective, they have to be at the forefront of the technologi$\mathrm{cal} / \mathrm{scientific} \mathrm{advancements,} \mathrm{otherwise} \mathrm{there} \mathrm{develops} \mathrm{a} \mathrm{time-lag} \mathrm{between} \mathrm{what} \mathrm{is} \mathrm{actually} \mathrm{needed}$ in the labour market and what is taught at the universities. This will result in students acquiring knowledge that is out-dated and no longer needed. 
As the rate of technological change increases, the rate of knowledge obsolescence (relevant knowledge) also increases. For example, the knowledge needed in the primary (agrarian/raw material) era is vastly different from the ones needed in Industrial era or the Service era.

We are now once again going through a period of increasing technological change akin to the first Industrial Revolution. In this transition from the Service era to an Information era, the Quaternary Sector is becoming increasingly dominant. The Quaternary Sector encompasses the knowledgebased parts of the economic activities such as R\&D, consulting services, information generation and transmission, education, and related knowledge-based activities.

The rate of information generation and accumulation in the Information era (its economic part: knowledge-based economy) is extremely high (as compared to the previous eras), resulting in an increasing knowledge base which has to be formalized and taught to the students. Since the rate of change is increasing rapidly, the time-lag between the knowledge creation and its transmission is becoming an important critical success factor for the economies of knowledge based societies.

Here the roles of the educational institutions become increasingly important. Not only do these institutions have to update their own staff, but they have to provide increasingly customized products to a larger customer base. Continuing education is also becoming a necessity for the majority of the knowledge workers, something that the educational institutions have to address.

All these changes and requirements present challenges that traditional educational institutions are poorly equipped to handle. What is needed is a new type of institution that can deliver the highly relevant and customized education that is needed by a knowledge society. And, it must deliver the products in a timely fashion that not only keep the products up-to date but are in a way that is convenient for the customer (student, knowledge worker).

\section{The Project Proposal, Roadmap and the Paper Structure}

The final results of digital ecosystems' projects (DBE and OPAALS) provided a suitable infrastructure and integrated theoretical framework for a distributed transactional model, as well as an open knowledge system, but customisation of such a model for educational use and the actual implementation were not part of the roadmap. Therefore we have proposed a new project focused on educational necessities.

Our primary studies have shown the inevitability of providing a collaborative environment between educational institutes on the one hand and the essentiality for offering an interactioncentred approach to knowledge sharing on the other (Siebes et al., 2007). Such a model has to offer a distributed interaction model to deliver an interaction-centered approach to knowledge sharing and introduce a stable Peer-to-Peer network for collaboration between educational institutes. This collaborative environment provides the facility to offer composed courses presented by different educational institutes and, as a result, the final degree can be awarded by a few universities (rather than a single university) with agreed educational standards and criteria.

In this paper we have focused on the technical feasibility of such a system. We have delivered an open framework for a distributed educational system where the consistency of the interaction model, dynamic topology, and the stability of the network have been approached and a stable model for each of them has been proposed. One may argue that a central organization should be responsible for quality assurance of courses offered by various universities. Furthermore courses should be checked to assure that they meet the British (or European Union's) educational standards and criteria. As we have limited the criteria to the technical feasibility of the model, we have to avoid the complexity of governance, possible qualifications' standard, and further social complications of the model (we will explore such repercussions in our future work). Meanwhile 
as the implementation of the system is in early stages, the actual execution of the model can be varied and further complications may arise in the actual application.

For propositioning our model, first we have introduced the concept of an "Open Education Network" (next section), which relies on the distributed interaction model (we have used our digital ecosystems transactional model as a primary conceptual model) and Open Knowledge Systems. In the next section, we have offered our primary framework that clarifies the general structure of the system in term of interacting with other knowledge-based systems. We have explored the details of the necessary components of such a system in "The Distributed Educational System" section, where we determine each components of such system, explain the role of each of them, their relationships and the potential network of the proposed system. At the end of this section two main challenges of the model are clarified: the consistency of interaction model and stability of the network.

Next three sections of the paper provide solution for these challenges. In "The Consistent Interaction Model", the detailed challenges of the interaction model and potential transaction model are introduced (which it is inspired by our previous works in OPAALS). The next section ("The Virtual University \& the Open Education Network") shows the actual practice of the model for creating integrated courses (virtual courses) through the collaboration of different educational institutes, the role of the network in that respect and reviews potential connectivity bottlenecks. "Increasing the Connectivity and Stable Infrastructure" presents a solution for a fully connected network, which overcomes the connectivity bottlenecks. At the end we conclude our paper with a brief conclusion and explain the future works of the project.

\section{Open Education Network}

The 'Social Network Services' combined with 'Open Knowledge System' can herald a new era for an Open Education Network (OEN). When a social network service is an online service, it essentially consists of a representation of each user, his/her social links, and a variety of facilities for users collaborating and sharing their thoughts (Boyd \& Ellison, 2007; Kelsey, 2010). An Open Knowledge System offers an environment for gathering, composing, and sharing the knowledge.

In contrast to the conventional education system, OEN can offer a distributed system, where individuals can create practical online learning systems to teach, share, and expand their knowledge and experiences by establishing a virtual university. One of the earliest relevant studies can be traced back to 1996, when Wellman proposed the idea of computer-supported social networks (Wellman, Salaff, Dimitrova, Garton, Gulia, \& Haythornthwaite, 1996). Weedman's study indicated "that there is a danger in distributed educational environments of providing insufficient support for the kinds of informal, exploratory conversations among peers that are important to intellectual work" (Weedman, 1999).

This proposal was the first indication of the interaction complexity. To address this, one can add online video streaming to increase the effectiveness of sharing knowledge in a collaborative project (Light, Light, \& Wright, 2000). A wider study by Butler (2001), showed that the actual complexity behind a social network service could be more than an additional one-off service (such as video conferencing). Furthermore, while the absence of centralized control (due to the distribution and openness of the network) increases the scalability, the sustainability of the network (in terms of the interaction model, network traffic bottlenecks, trust, identity, etc) can raise serious questions for the feasibility of such a model (Razavi, Moschoyiannis, \& Krause, 2007a). Therefore we are dealing with a fully distributed environment, where the virtual education platforms 
due to their methods of interactions (text ${ }^{1}$, offline videos ${ }^{2}$, streaming ${ }^{3}$, etc), may create regular bottlenecks and threaten the environment (particularly the network).

\section{Digital Ecosystems as an Example of a Distributed Conceptual Model}

A suitable European model for such a distributed system has been introduced by the European Commission (Nachira, 2002) ${ }^{4}$. Developing this conceptual framework has not been limited to Europe. The digital ecosystems and Business Intelligence Institute in Australia (DEBII), a leading research institute on digital ecosystems studies, has already developed and constituted digital ecosystems. One of the best descriptions of the Digital Ecosystem can be found in Chang and West's definition:

"There are four essences of ecosystems: (1) Interaction and engagement (2) Balance

(3) Domain clustered and loosely coupled (4) Self-organization”. (Boley \& Chang, 2007)

The loose coupling and local autonomy of participants, in the absence of centralized control, will result in distributed coordination, which creates a fully distributed environment (Razavi et al., 2007a). As coordination of the interaction has been performed and controlled by each participant independently, the transaction/interaction can be triggered, executed, and committed by any participant/agent (Krause et al., 2008). In addition, a self-organizing mechanism should be able to provide stability of the network against bottlenecks and failures (Razavi, Moschoyiannis, \& Krause, 2008).

In digital ecosystems, each business transaction is the result of peer-to-peer interactions between several nodes in a network in order to reach a specific target. The transaction will be expressed by its context, which can be represented in an xml file, and shows the order and relationships of services in a transaction (Razavi, 2009). Therefore the transaction context clarifies the orchestration of services. Because each of these services has been provided by different participants of a transaction (for example an airline, a hotel, and a Minicab-company can be involved in a simple travelling transaction), they will be provided at the network location of their providers (ex: the airline computer system, the hotel reception pc, and the Minicab-company's laptop) and their relationship, including sharing results, ordering dependencies between services, etc., shows the necessary interactions between these participants. Therefore we are dealing with a service-oriented environment, which includes several overlapping networks of transactions as a result of fully distributed peer-to-peer interactions (Razavi, 2009).

Based on these characteristics, it will be adequate to consider an Open Education Network as a model for our digital ecosystems. To implement this (virtual university and OEN as its infrastructure), we can apply and extend the results of EPSRC and European commission projects.

\footnotetext{
${ }^{1}$ This includes Internet forums, message boards, interactive rating systems, online chat or any sort of conversation in the form of 'thread' of texts.

${ }^{2}$ Off-line interactive videos are popular in most video sharing web sites, when one of users release a multi-media descriptions or presentation (in form of off-line video) about a subject and audience will response or challenge the concept by providing detailed visual presentation (in form of off-line videos).

${ }^{3}$ Streaming has been presented in the form of interactive live videos broadcasting, videos and voice conferencing, interactive live presentations (Oracle, Microsoft and many software companies use this method for their online courses or promoting their products) and many other new video and voice streaming services.

${ }^{4}$ This framework has been reflected in several European projects practically; DBE (Digital Business Ecosystem 2002-2006; DBE/ EU FP6-IST/ Contract No 507953) and OPAALS (2006-2010; OPAALS/ EU FP6 NoE/ Contract No 034824).
} 


\section{Open Knowledge Systems}

The potential knowledge available through the Internet is immeasurably large. Siebes and Dupplaw (Siebes et al., 2007) have described the aim of sharing knowledge in an open knowledge environment as "to obtain knowledge appropriate to the activities in which each consumer wants to engage, while maintaining free and (adequately) reliable connections between suppliers and consumers". In contrast to the traditional data-centered approach, the interaction-centered approach to knowledge sharing relies on the interaction models in a service-oriented architecture. In a service-oriented architecture, web applications supply information by Web services accessed through various forms of application interface (Curbera, Duftler, Khalaf, Nagy, Mukhi, \& Weerawarana, 2002). When the purpose of service-oriented computing is to facilitate, invoke, and execute services in a distributed, scalable and interoperable manner, the Semantic web tries to provide the feasibility for (automatically) locating and composing such services (Sycara, Paolucci, Ankolekar, \& Srinivasan, 2003).

In this paradigm, the semantic web tries to produce information through a complex program (web application and their interfaces as web services), which may interact in complicated ways (Siebes et al., 2007). Traditionally the interaction models of web services rely on the composition of web services which in turn rely on service descriptions. Web service descriptions can simply be descriptive such as WSDL $^{5}$ (Christensen, Curbera, Meredith, \& Weerawarana, 2001), which simply clarifies inputs and outputs, or semantic based such as WSDL-S ${ }^{6}$ (Sivashanmugam, Verma, Sheth, \& Miller, 2003) and OWL-S ${ }^{7}$ (Martin et al., 2004).

One of the practical models for Open Knowledge Systems has been proposed by Siebes et al. (2007), where they describe an operational system that uses models of interaction as the focus for the knowledge exchange. Their implementation adopts a peer-to-peer architecture, making some assumptions about centralization of knowledge sources, discovery and specifically interaction control. This acts as a crucial bottleneck and reduces the scalability of their model. The OEN has ambitions to work on larger-scale for providing facilities for a global Virtual University. The proposed Digital Ecosystem infrastructure (Razavi, Moschoyiannis, \& Krause, 2007b; Krause et al., 2008) appears to be a strong candidate to extend Siebes' model. At the same time, the distributed coordinator model of DE (Razavi et al., 2007a) can be used instead of the interaction controller.

\section{A Primary Framework}

The main purpose of an extensive Virtual Education System is to provide a framework within which participants not only can present the traditional data-centered approach for expressing their knowledge system to the end users, but also be able to offer the interaction models for collaboration amongst each other. Implementation of this paradigm is made more feasible by the use of the service-oriented architecture and conceptual frameworks such as Web 2.0

\section{Local Content and Knowledge Consumption}

The conventional model for an electronic educational system relies on the traditional data-centred knowledge approach (Siebes et al., 2007), where the knowledge services can be accessed through

\footnotetext{
${ }^{5}$ WSDL (Web Services Description Language) is an XML-based language that provides a model for describing Web services.

${ }^{6}$ WSDL-S extends standard WSDL to include semantic elements which should improve the reusability of Web Services

${ }^{7}$ OWL-S is an ontology, within the OWL-based framework of the Semantic Web, for describing Semantic Web Services.
} 
a standard interface. This standard interface can be a ubiquitous web page such as presented in Figure 1.

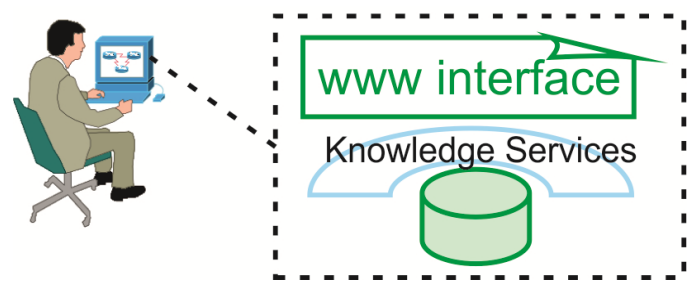

Figure 1. A conventional knowledge system

Unsurprisingly such a system is dependent on a single knowledge framework and can simply be managed by any Content Management System (Addey, Suh, Ellis, \& Thiemecke, 2002), which usually is made up of a front-end editor for inputting content, a back-end system for storing the content, and a template mechanism to get the content onto the web site. Such a model may be unable to interact with another knowledge system to share its contents or collaborate with another system to add additional meta-information to its current contents or produce new knowledge.

\section{Local Coordination and Interaction Model}

In contrast to the long-established knowledge systems, which rely on a data-centered approach, the modern knowledge based systems offer an interaction model for sharing knowledge between different educational institutions. Such a model provides the framework for coordinating the interaction. Figure 2 shows a simple demonstration of this paradigm.

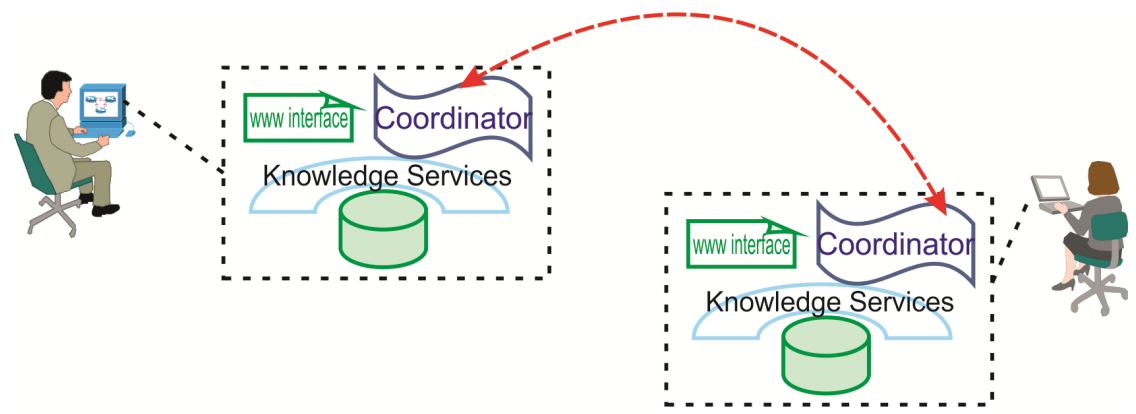

Figure 2. Contribution and coordination in knowledge systems

Despite the apparent simplicity of Figure 2, there are some complex issues that must be addressed. In the first place, a consistency model has to be used to provide a framework which assures the consistency of data (avoiding over-writing transaction data or reading inconsistent data items). Regardless of the supported consistency model by the coordinator, the interfaces for sharing the knowledge, promoting, discovery, and creating the new types of courses through the conventional interfaces have to be addressed.

\section{The Distributed Educational System}

Service Oriented Architecture (SOA) is the tangible architectural approach of SOC and is mainly applied when multiple applications running on different technologies and platforms need to communicate with each other (Singh \& Huhns, 2005). In this way enterprises can mix and match services to perform their transactions with minimal programming effort. SOA is a way of reorganizing software applications and support infrastructure into an interconnected set of services, 
each accessible through standard interfaces and messaging protocols. The basic SOA is not only about architecture of services, but also about the interaction between three kinds of participants: the provider; the discoverer; and, the requestor. These interactions involve 'publish', 'find' and 'bind' operations (Papazoglou \& Georgakopoulos, 2003).

In a typical service-based scenario a service provider hosts a network-accessible software module (an implementation of a given service), where the service provider defines a description of the service and announces it to a client or service discovery agency through which a service description is published and made available. The service requestor uses a find operation to retrieve the service description (typically) from a discovery agency, i.e., a registry or repository like Universal Description, Discovery and Integration (UDDI), and uses the service description to bind with the service provider and invoke the service or interact with the service implementation (Papazoglou, Traverso, Dustdar, Leymann, \& Kramer, 2006).

In our educational system, the initial state of the system is based on a data-centered approach, where the knowledge of each provider has been stored locally and the actual system has local autonomy. The theoretical design of each platform should respect the autonomy (of whom or what) and avoid interfering with the actual representation of the information. In this way the information will be accessible though the implemented services by the educational system. Conceptually we consider this platform to be a local agent. The primary needs of this agent are to find, publish and bind its services. To address these needs it requires:

- a 'Local Repository', where it keeps its local educational services and a 'Global repository' (which keeps the information about other participants' knowledge centres and their services);

- an interface for publicising the description of its services for other participants (it can be kept by submitting in their Global repository);

- an interface for discovering and updating the information about other knowledge centres (for updating its Global Repository, according to the other Participants' knowledge). Figure 3 shows such a model.

\section{Education Centre Conceptual Design}

If each Education centre is considered as an independent platform, we can then assume that a local agent with six conceptual components can cover the 3 main operations of SOA in an autonomous manner (Figure 3). We call this agent the "Knowledge Agent";

\section{Component 1: Local knowledge centre}

The kernel of any educational centre is its 'Local Knowledge Centre', i.e., a repository for keeping the description of (and the method of accessing) its educational services. The necessary interfaces to this repository are the following: an interface for the administrator to manipulate its services such as add, update or delete; an interface for promoting (publishing) its knowledge for other Knowledge Agents (Education Centres); and an interface for interacting with other Knowledge Agents in terms of a transaction.

\section{Component 2: Local knowledge manipulator}

This component is to provide the user/administrator with an interface to manipulate the local knowledge directly. This interface can be a standard CMS' web interface.

\section{Component 3: Global knowledge centre}

As has been mentioned at the beginning of this section, each participant (knowledge agent), should have a global repository that keeps information about the other participants' services and 
the methods of accessing them. This information can include a simple description of services or any type of metadata which can help the organisation to effectively use and compose their services with its local (native) services. In contrast to the general repositories in SOA, this repository is customised for each Knowledge Agent and is not centralised (or controlled by an external company/organisation).

\section{Component 4: Knowledge investor}

The view of each Knowledge Agent of other Education Centres and their services is reflected in its Global Knowledge Centre. A "Knowledge Investor" component allows the Knowledge Agent to investigate potential partners (other Knowledge Agents / Education Centres) and new services for adding to its Global Knowledge Centres.

\section{Component 5: Knowledge promoter}

Each Knowledge Agent should be able to promote its services, offer its remote services and provide for the possibility of composition of its local services with other Education Centres. A "Knowledge Promoter" component offers this facility, by connecting to a "Local Knowledge Centre" component. It will then have the latest local services and their descriptions which it can use to update another Education Centre.

\section{Component 6: Local coordinator}

The Local Coordinator plays the main role in interactions between different Knowledge Agents. It provides a transaction model for service compositions between different Knowledge Agents. It must offer a distributed transaction model which is consistent and recoverable. In the next section we explain the complexity of such a model.

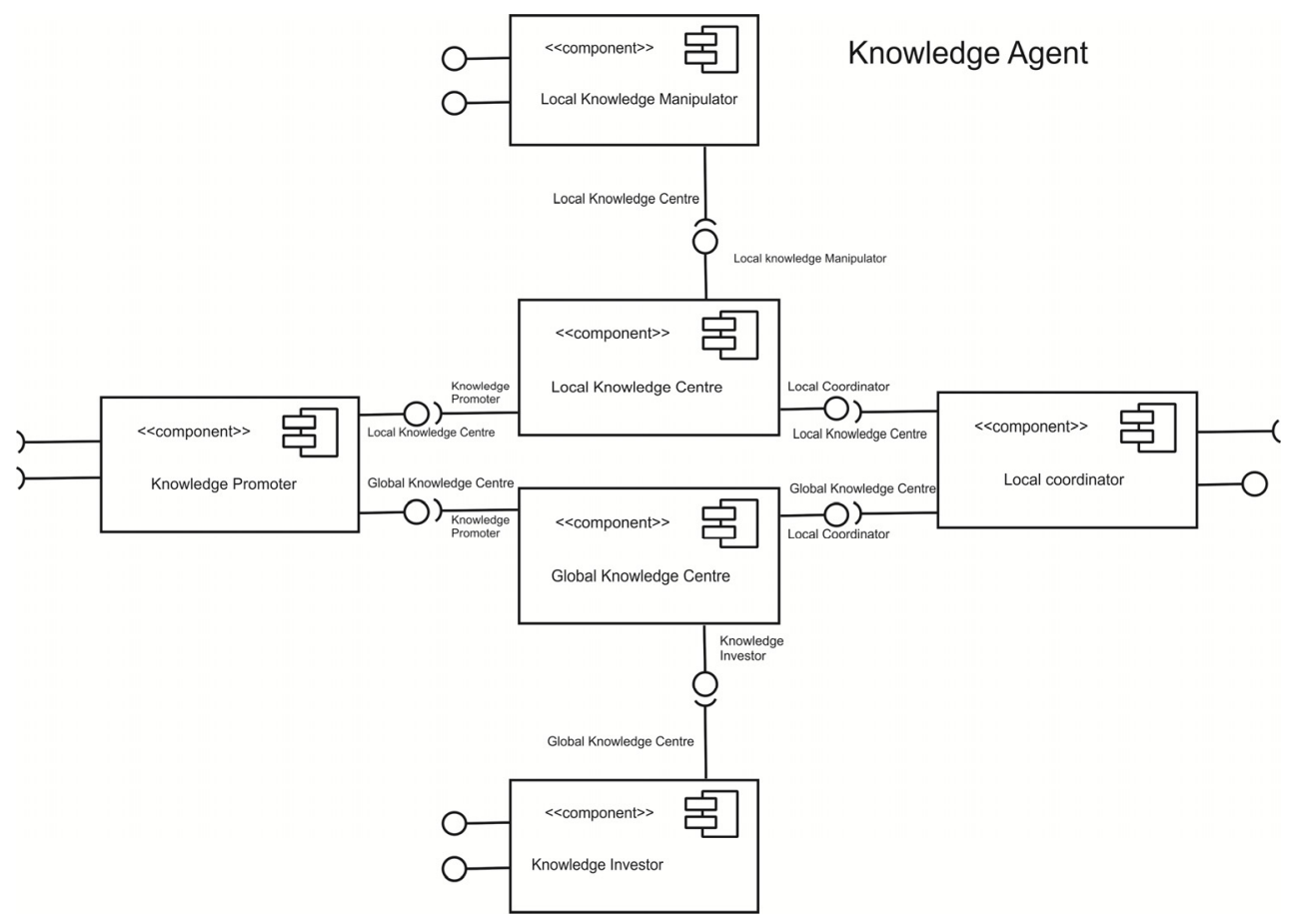

Figure 3. A conceptual model of a knowledge agent 


\section{Open Educational Network: Consistency and Sustainability}

As can be seen in Figure 4, any Knowledge Agent (KA) can have different types of connections to other KAs (Education Centres). A KA can promote its local services (information in its Local Knowledge Centre) or its information related to the Education Centres (its stored information in its "Global Knowledge Centre"). This can be done by using the interfaces of the "Knowledge Promoter" and connecting it to the other KAs' Knowledge Investor interfaces (see Figure 4). Similarly, the interfaces of a Knowledge Investor of a KA (KA 1 in the Figure 4) can connect to the Knowledge Promoter of another KA (KA 3 in Figure 4) to use the latest information stored in that Knowledge Agent (KA 3). Furthermore, the actual transaction (service compositions or simple interaction between KAs in terms of the remote accessing to each other services), can be done by their Local Coordinators (KA 1 and KA 4 in Figure 4).

The main aim of this model is to propose a loosely coupled solution based on the context of current educational systems (their e-learning systems, online courses, and services), which can facilitate collaboration and composition of their services. In this way challenges can be categories in two related areas: transactional challenges and network related problems. The transactional challenges can be addressed by a coordination framework which offers a consistent solution for interaction and composition of services while at the same time, the traffic complexity, network fragmentations, and platform failures should be handled.

Such a solution should provide a distributed coordination model with full consistency and recoverability properties. In addition, the solution must support a dynamic topology that continuously adapts to reflect the actual usage of the network in terms of transactions. The other important property is resistance against fragmentation. Moreover, the proposed solution ought to avoid a central point of command and control and should not suffer a single point of failure. 


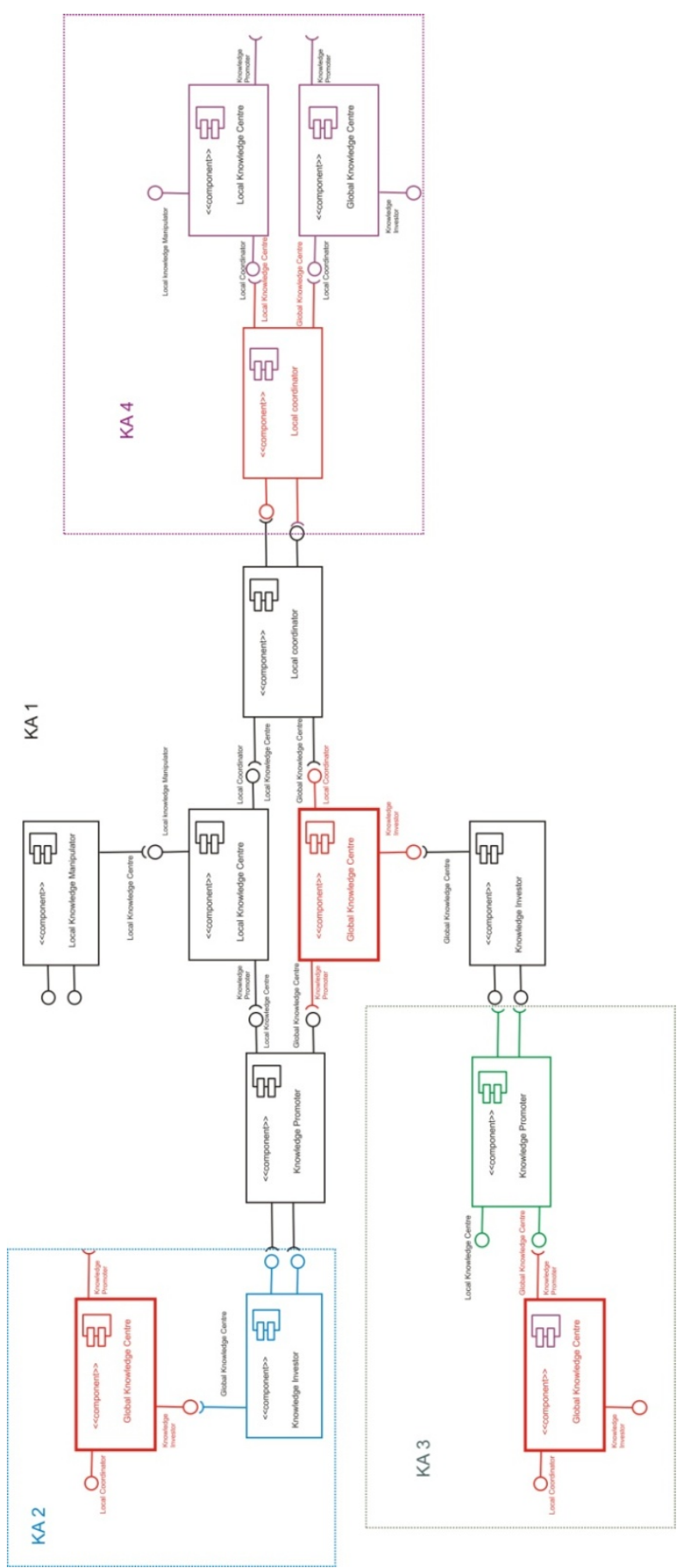

Figure 4. Connections and contributions between knowledge agents

When components such as the knowledge investor and knowledge promoter are dealing with the network challenges, such as increasing connectivity during the interaction with other educational systems, the local coordinator should take care of the distributed transaction model for service composition and provide the integrated on-the-fly services. Figure 5 shows an example of such a network. In the next section we shall discuss these problems and our solutions in more details. 


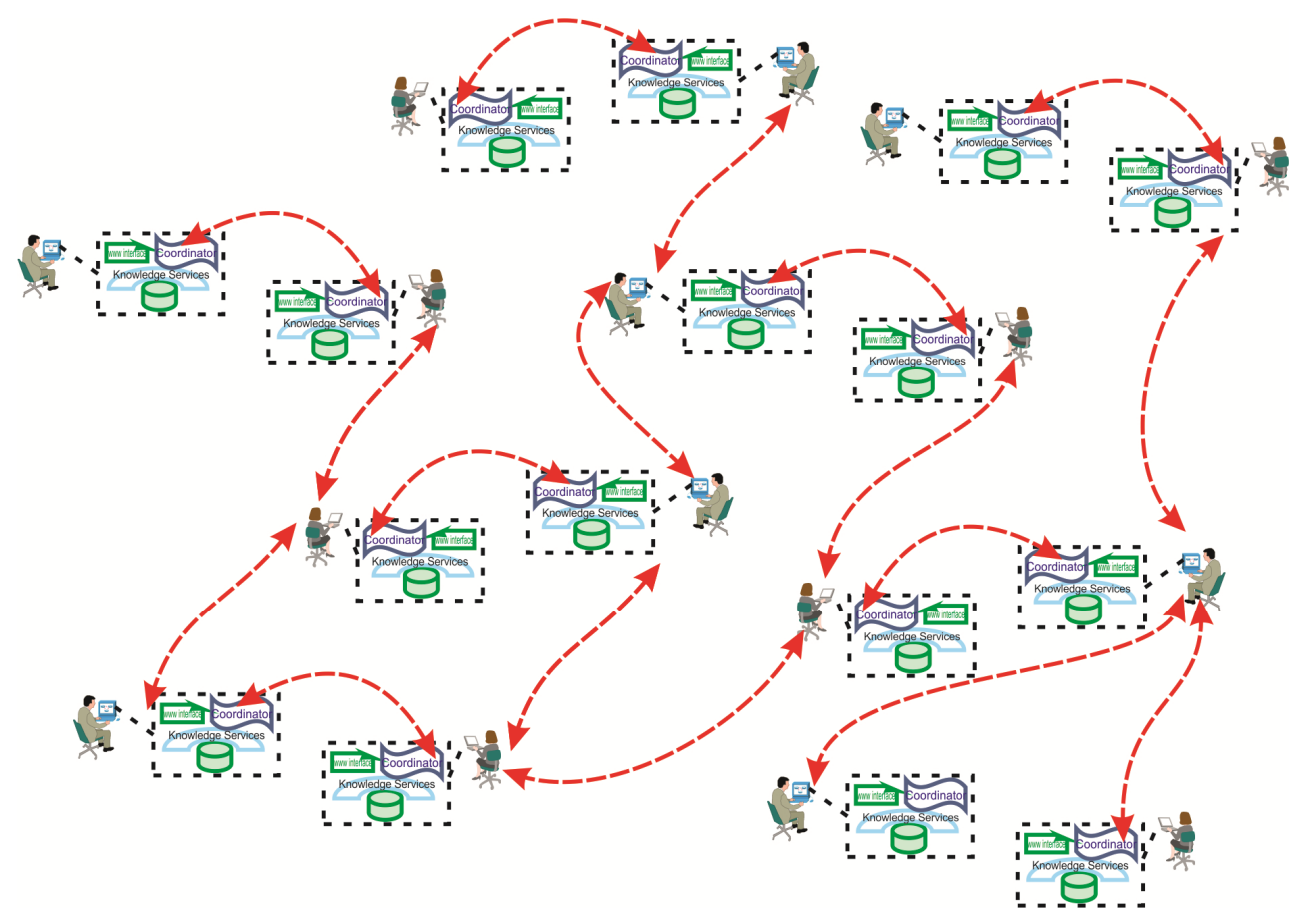

Figure 5. An Open Educational Network

\section{The Consistent Interaction Model}

The transactions between Knowledge Agents are supposed to use service-oriented architecture to invoke and execute services in a distributed, scalable, and interoperable manner. The conventional definition of a transaction (Date, 2003; Gray \& Reuter, 1993) is based on Atomicity, Consistency, Isolation, and Durability (ACID) properties. While the ACID properties provide a good foundation for conventional database applications, they can present unacceptable limitations and reduce performance in advanced applications.

These limitations have been traced to three specific areas in conventional transaction models (Elmagarmid, 1992; Haghjoo, Papazoglou, \& Schmidt, 1993; Kakeshita \& Xu, 1992; Moss, 1985): Long-lived transactions; Lack of partial results; and Omitted results.

The necessity of change is derived from the nature of modern applications (such as business applications and CAD projects) (Elmagarmid, 1992). For example, the specification of a transaction may allow it to be completed over a period of hours or even days (a "long-lived transaction"). In addition, the obligation for cooperation between transactions can be specified in a business process rule (a requirement for availability of "partial results"). Finally, the instability of the Internet environment can define a new requirement for keeping important results even when the connection between two platforms is lost ("omitted results").

\section{ACID Properties and Challenges}

Although a conventional transaction model could provide solutions for a huge range of applications (such as databases and ACID transaction based applications), its limitations may cause serious problems for advanced applications (Elmagarmid, 1992). CAD applications, business-tobusiness interactions, distributed computing, and most enterprise applications deal with transactions that have long durations. Based on the conventional model, these transactions will lock resources and limit access to them (this means that other transactions cannot access the data before the transaction commits). This waiting time will not only reduce the overall performance of the 
system dramatically, but will increase the probability for deadlock and violate the concurrency laws (the overall performance of concurrent transactions should not be less than a serial execution of them) (Gray \& Reuter, 1993). This transactional problem is addressed by 'Long-lived transactions'.

For solving the Long-lived transaction problem, Eliot Moss (1985) proposed the "Nested Transaction". The concept of "Nested Transactions" has relaxed the atomicity property of conventional transactions (A from ACID properties). Instead of traditional atomic transactions, "Nested Transaction" offers a hierarchical structure where a transaction divided to levels of sub-transactions in a tree-structure. In this way Moss tries to apply the consistency and atomicity properties in a finer level (sub-transaction) to reduce the probability of long-lived transaction problems. However, many problems still remained unsolved and Moss' Nested Model may not be applicable for several areas (especially when releasing partial-results between Nested-Transactions is necessary).

Different non-conventional transaction models were subsequently derived from the NestedTransactions model, which changed the face of the transactional world. In the Nested transaction model, each transaction can have a tree structure (including many sub-transactions) and each node can share its results with the others in the same transaction. However, one Nested transaction cannot share its results with any of the other nested transactions. Therefore each nested transaction still was atomic and isolated as far as other nested transactions were concerned and hence the ACID properties were still applied, although at a different level.

One of the important requirements in the distributed computing domain is the ability to access the information before a transaction has finalised (commit). In the conventional Nested Transaction model (Moss model), in order to maintain transaction consistency, an internal limitation has been applied and the interaction (sharing the results) is available only internally within a transaction. This means that only the parent of a sub-transaction can receive the results of its child and all other transactions have to wait for commitment of the main transaction. In some applications, this can create a bottleneck when other transactions need the result of that particular transaction. This will be more complicated when we are dealing with several concurrent transactions and they need some results from each other. In these cases, the logic of an application may require access to the results of uncommitted transactions but the transaction constraints do not allow such access. This problem arises through the unavailability of 'Partial Results' (Elmagarmid, 1992; Haghjoo et al., 1993; Kakeshita \& Xu, 1992).

In addition, based on conventional nested transactions, if a transaction is (for any reason including a simple failure) aborted then all of its sub-transactions must also abort. This means that all of the intermediate results produced up to that point must be rolled back to the initial values. In most cases, the transaction abortion is followed up by a restart (this means another try to execute an unsuccessful transaction). Based on this model, there is no differentiation between full abortion (as a result of a failure) and restart. In both cases, all sub-transactions will be rolled back and will be redone. This will cause a huge overhead and waste the resources of the environment (where successful operations could be kept until the transaction restart). This problem is known as 'Omitted Results' (Razavi et al., 2007a; Razavi, 2009).

\section{Educational System's Transactions}

The fundamental problem for long-running transactions is the necessity for sharing objects between the concurrent transactions. On one hand the concurrency control should support the concurrent activities. However, update of an object when it is accessed by another transaction can violate the consistency of the environment. Therefore concurrency control should be able to provide a consistency model and an acceptable level of isolation. Furthermore, recoverability of a transaction relies on these limitations too. When transactions are prevented from accessing incon- 
sistent objects of other transactions, each transaction will be recoverable. But applying conventional isolation theorems ${ }^{8}$ will reduce the performance of the environment significantly (as longrunning transactions have to wait for commitment of each other) and at the same time, they will increase the probability of deadlock (Razavi, Moschoyiannis, \& Krause, 2007c).

Through many different approaches to supporting concurrent execution of transactions, a consensus on the use of locks as a preferred solution has emerged (Bernstein, Hadzilacos, \& Goodman, 1987; Gray, 1992; Gray \& Reuter, 1993; Ramakrishnan \& Gehrke, 2003). Other than implementing locking, we should take into account that concurrent execution should not have lower throughput or much higher response times than serial execution. The second law of concurrency control (Gray \& Reuter, 1993) is to avoid high computational overheads. At the same time, the traffic complexity can create bottlenecks on different nodes on the network.

\section{The Virtual University \& the Open Education Network}

\section{Virtual Courses and Virtual University}

Theoretically an OEN can offer the integration of different courses where each course may be presented by different educational institutions. Then, an anthology of these courses can provide virtual courses and virtual qualifications. The qualification(s) can be presented (awarded to students) by a few educational institutions rather than one university. Consequently universities and different institutions can offer a variety of degrees, even when they do not have all the facilities for doing it by themselves alone. They may rely on other partners' expertise as well as providing them their own unique knowledge. In this way, on one hand they will be able to offer other edu-

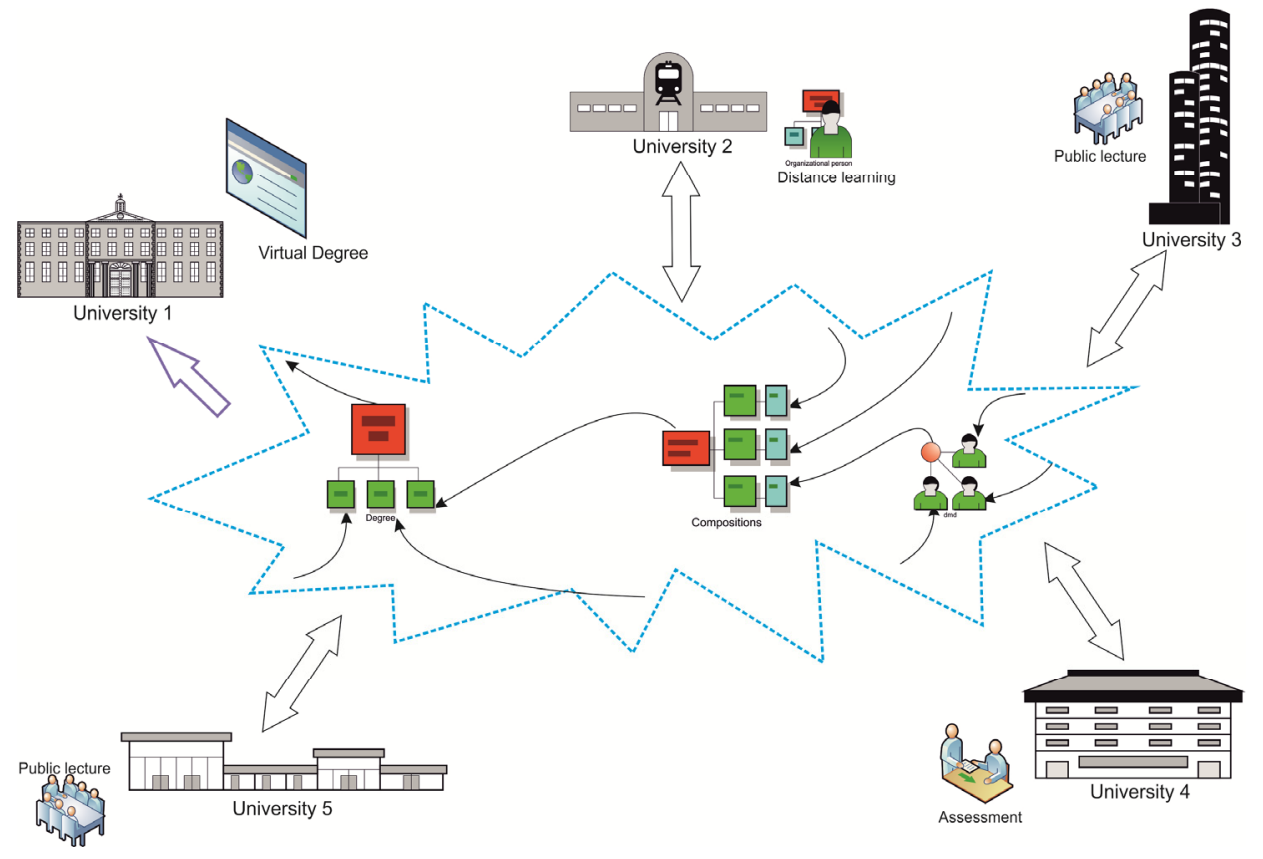

Figure 6. A simple scenario for Virtual Degree

\footnotetext{
${ }^{8}$ The isolation theorems are the classic method for showing the correctness of the transactional environment. The main result of the study in isolation can be summarised as follows: a serial execution of transactions is always correct, when each transaction follows the commit or full rollback of the other one.
} 
cational partners their educational services and earn some extra income, while on the other hand, they can offer a greater range of expertise which can meet their geographical necessities. Figure 6 shows such a scenario.

As can be seen in Figure 6, as well as a consistent interaction model, a dynamic network infrastructure plays a major role in this model.

\section{Stable Open Education Network}

The aim of the Open Education Network is to provide a stable environment (network) while facilitating interaction (different levels of transactions) between participants (educational institutions). For this purpose, we distinguish interactions/transactions from services (and service providers) by considering Virtual Private Interaction Networks (VPINs) and Virtual Service Networks (VSNs). These two virtual networks are optimized individually and in respect to each other. The effect of one on the other can supply us with stability, failure resistance, and smallworld characteristics, on one hand, and durability, consistency and sustainability on the other. The proposed network design has to have a dynamic topology that adapts itself to changes in the Knowledge-based models and availability of contents and services of participants.

A number of different views exist on the development of a sustainable network, from that of a collaborative environment for long-lived transactions, to a software infrastructure for open ebusiness transactions, to the continuous creation of new business model categories and instances. All these different views can challenge the current infrastructure of our software world. At the same time, the telecoms industry is moving towards Next Generation Networks (NGNs), and this comes with yet another view of services and applications; the so-called Next Generation Services (NGSs) (Daho \& Simoni, 2006). Our approach is to try to leverage these developments in creating a stable Open Education Network which supports dynamic contexts for distributed long-lived transactions as well as conventional interactions.

Current models which provide self-management capabilities at the service level (Gray, 1992; Kristiansen, Hansen, \& Licciardi, 2008; Li \& Mohapatra, 2004; Martini, Baroncelli, \& Castoldi, 2005 ) and Quality of Service (QoS) at the virtualization levels (Daho \& Simoni, 2006) can be seen to satisfy the primary requirements of such an Open Education Network. However, there is little evidence that they can insulate the collaborative activities of long-lived transactions from failure in the face of a highly dynamic environment.

As a result, keeping the necessary information for coordinating long-lived transactions (Razavi et al., 2007a) can be rather challenging, but also the probability of fragmentation in the network cannot be averted. This can have severe consequences in a business environment, since fragmentation in the network of course directly affects the number of failed transactions (Razavi et al., 2007c). In the next section we outline the main characteristics of such a network and clarify the necessary virtualization of the model.

\section{The Connectivity and Bottlenecks}

The purpose of an Open Education Network is to enable networked organizations (educational institutions) to engage in distributed transactions that realize their core educational activities. As was presented in the previous section, in terms of interactions, this means stronger interconnectivities for VPINs. This is achieved when a transaction's participants can avoid failure at the supporting network level and/or alternative paths are reachable whenever service unavailability is experienced or failure occurs in one of the participants in alternative scenarios.

This may lead us to increase the local connectivity in the interaction level, but the effect and side effect of changes will direct us to take into account a measurement for stability and apply any 
increases in terms of this measurement. This provides a dynamic and extensible method for creating a stable network that emerges through the long-running transactions that correspond to the collaborative activities between participating organizations. Meanwhile, providing a level of virtualization for applying OEN conceptual foundations seems necessary. One of the important characteristics of OEN is its dynamic nature. The network ontology, which represents states of network devices and servers and other infrastructure resources in a logical virtual construct, may change according to network traffic complexity and transformations.

By their very nature, participants of an OEN would deal with multimedia material, which can be streamed online. As a result, there is a considerable amount of traffic. Furthermore, the direct regional effect (such as institutions' opening hours, peak access time to the system, time-table for live streaming) is another property that needs to be taken into account in an OEN. These are some of the factors that make it necessary for the network topology of such an environment to be able to adapt itself dynamically (dynamic topology).

For this reason the virtualization in our model is slightly different from that found in the general proposed models (such as Daho \& Simoni, 2006; Gray, 1992; Kristiansen et al., 2008; Li \& Mohapatra, 2004; Martini et al., 2005). The core activities of participants (long-lived transactions or conventional atomic interactions) are at the top level of the model: such activities in terms of transactions which should be distributed and recoverable (in term of any type of failures). In previous work, similar transactions have been introduced (Razavi, 2009). The result of the interaction between participants (different educational institutions) of a transaction, which comes down to the composition of the corresponding services, provides a virtual connection. Keeping this temporary network as a virtual network can be useful for the design of the underlying network. This virtual network, shown as the first conceptual level of virtualisation in Figure 7, is private between transaction participants, and hence the term Virtual Private Interaction Network (VPIN).

The second conceptual level of virtualization is concerned with links between participants (education service providers and actual participants of transactions) which provides the structural materials for an interaction (transaction). These structural materials are 'services'. We call such a virtual network a Virtual Service Network (VSN). Figure 6, shows the virtualisation levels of the networks.

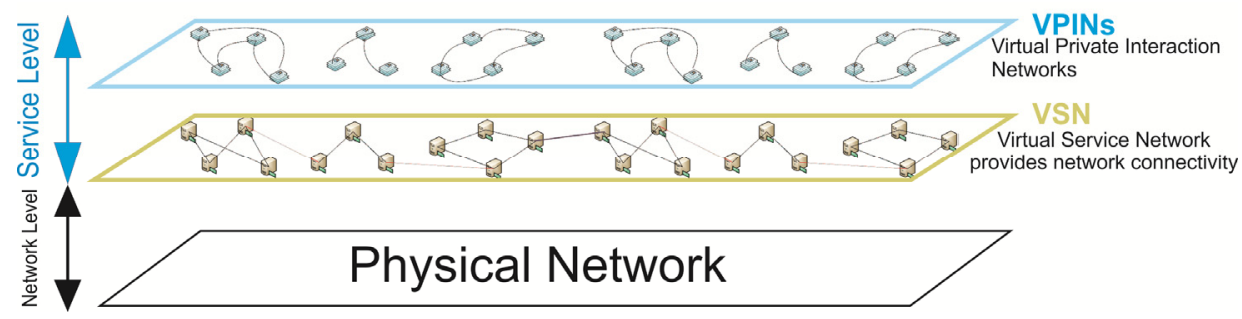

Figure 7. Network Virtualisation

Even though participants may be engaging in more than one transaction at a time, a VPIN is typically a fragmented (island) network that connects the participants of a specific interaction. Therefore, nodes in VPINs are typically in the same domain (or semantically related domains). For this reason, VPINs potentially may improve the 'cluster coefficient ${ }^{9}$ ' of the lower level (VSN) and in exchange VSNs can warranty the diversity for VPINs (by providing reliable connectivity which makes alternative paths/scenarios feasible for a disconnected VPIN).

\footnotetext{
${ }^{9}$ Generally we may define cluster coefficient for a measurement for the neighbourhood connectivity of the node. If the neighbourhood is fully connected, the clustering coefficient is 1 and a value close to 0 means that there are hardly any connections in the neighbourhood.
} 
This may protect the transactions against failure - using alternative paths and scenarios to avoid the costly abortions of long-running transactions is feasible, as shown before. This is referred to as forward recovery (Razavi, 2009). It can be seen that one of the most important characteristics the VSNs should provide is 'connectivity' - ensuring that there is a network of interconnected nodes.

\section{Increasing the Connectivity and Stable Infrastructure}

Increasing the connectivity between participants of an interaction prevents certain types of failures in the transaction, predominantly those which are the result of the network disconnections. At the same time, alternative scenarios in terms of alternative service compositions (this will avoid the failures in term of interaction scenarios) rely on available connectivity between alternative paths (between different participants) in the network. This means increasing VPIN interconnectivity helps to provide a better chance for forward recovery (recall the consistent interaction model section) and, as a result, avoids a full recovery even when some participants failed to provide their services. Before describing how VPIN interconnectivity is dealt with in our framework, we present the general mechanism for link replication in the local software agent of each participant.

Normally the connections (links) to other participants of the Open Education Network will have been established by the 'Global Knowledge Centre', where the addresses of other knowledge Agents (participants) and the descriptions of their services have been kept. For inserting (or modifying) a new participant to the repository and its services, the 'Knowledge Investor' component will be involved. For introducing the participant to another participant the 'web service promoter' will be used.

It can be seen that for increasing the connectivity we use three components of the componentbased design of each participant. Figure 4 shows the relationships between these components of three participants (their software agents). Knowledge Agent $\mathrm{B}$ (' $K A B$ '), receives all of the connections of Knowledge Agent A ('KAA') through its 'Knowledge investor' when Knowledge Agent A (' $K A A$ ') provides them through its 'Knowledge promoter'. Similarly, Knowledge Agent $\mathrm{B}$ ('KA B') provides its connections (links to the other participants) to the 'Knowledge investor' of Knowledge Agent C (' $K A C$ '). We call this procedure Link Replication. It is important to mention that it is possible to have partial link replication where there is no need to replicate or pass to other participants (Knowledge Agents) all of the connections of a given participant.

\section{Fully Connected VPIN and Digital Ecosystem}

As we mentioned in 'The Distributed Education Systems' section and the previous section (last subsection), one of the significant risks for the VPINs of a digital ecosystem (transactions of the network) is disconnection between participants of an interaction which amounts to low connectivity inside the VPINs. By repeating link replication in each participant, within a limited time, all participants in a VPIN will be connected together. As a result we will have a fully connected $\operatorname{VPIN} P_{t}\left|F_{t}\right|-1$.

Where this seems like an ideal solution for each VPIN the result can be devastating for the digital ecosystem and consequently the majority of transactions could be failed. As a digital ecosystem is a connected network through its transactions (Razavi, Moschoyiannis, \& Krause, 2008), the VPINs have overlaps on some of their participants (there are some intersections between different VPINs' participants) and the network should at least follow the power-law distribution degree (Barabási \& Albert, 1999).

Based on the power-law distribution degree, the connectivity of the network will grow in a way that a few participants (nodes) which have the most interaction can provides the most possible 
links (and keep the stability of the network). Typically a network with power-law distribution degree has a few nodes with lots of links (they have called hubs) and the most of nodes has a few links to the other nodes. This means if nodes with a few links nodes (which are the majority of nodes) fail, the network will not fragmented but failing hubs may cause fragmentation in the network (Razavi et al., 2007b).

Now we apply link replication to increase the connectivity of the network, this will reduce to possibility for fragmentation, which means it increases the stability of the network. If we express the $i t h$ transaction by $t_{i}$, and its transaction participants by $P_{t_{i}}$, then its VPIN of transaction $t_{i}$ can be presented by $\operatorname{VPIN}_{i}\left(t_{i}, F_{t_{i}}\right)$. Now if we apply link replication for each VPIN, the number if links of each participant in the VPIN will be

$\left|F_{t}\right|-1$

Therefore a participant $R$ which is involved in several transactions from $t_{m}$ to $t_{n}$ $\left(\left\{t_{t} m, \cdots, t_{t} n\right\}\right)$,

will have up to

\section{$\sum_{-m}^{n}\left|F_{t_{i}}\right|-(m+n)$}

links.

Therefore by applying link replication in this way these small numbers of stable participants are involved in most interactions and will have a very large increase of links.

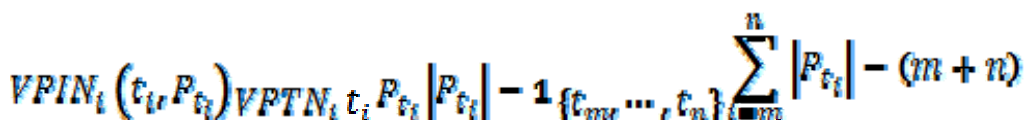

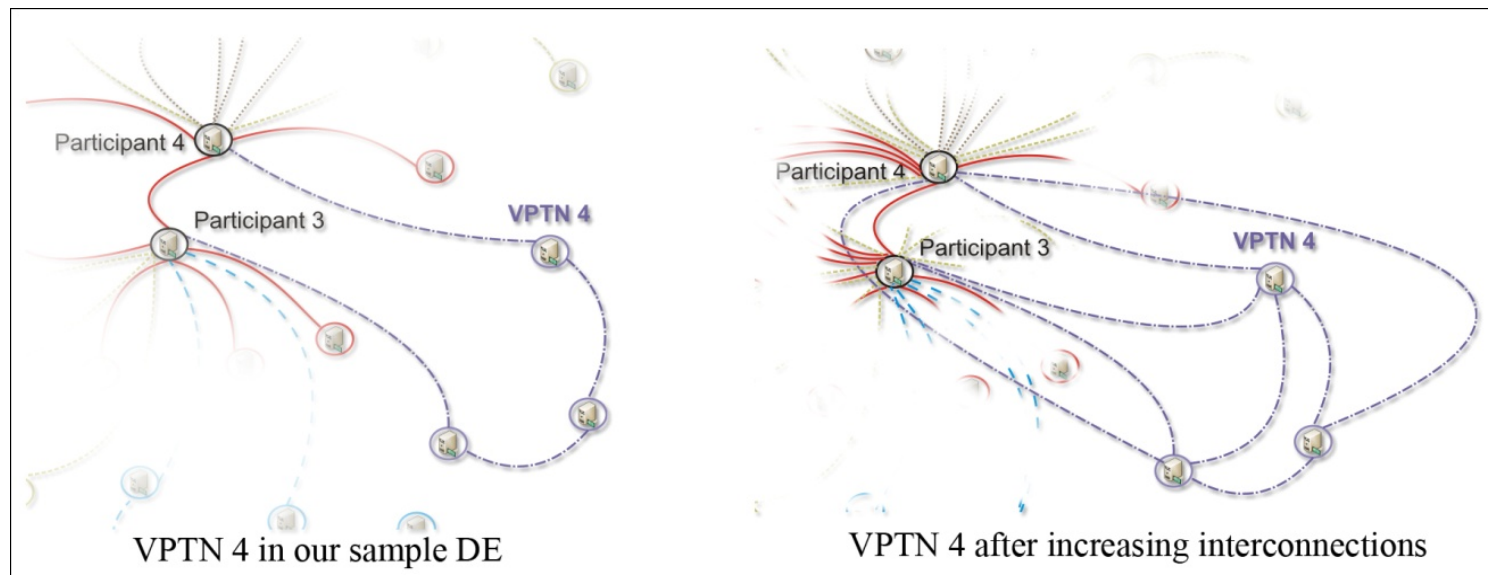

Figure 8. Fully disconnected VPINs

On the left side of Figure 8, VPIN4 from the sample digital ecosystem is shown, and on the rightside we can see the result of link replication on all participants of the VPIN4. As link replication in a similar way has been applied for all other VPINs, participant 3 and participant 4 which are involved in several transactions face the large increases of links which can bring traffic complexity. While the link replication itself seems quite useful for increasing connectivity, the way and 
on which participant it is applied can be crucial for the general performance of the digital ecosystem (and even each VPIN). By considering a consistent interaction model (see previous section on this topic) and applying link replication, the feasibility of an Open Education Network has been shown and virtual university concept can use most of small-world properties of a scale-free network (Barabási, Albert, \& Jeong, 2000).

\section{Evaluation}

In term of evaluating the model, as the interaction model has been inspired by OPAALS (has been reviewed in "The Consistent Interaction Model" section), we focus on the proposed Peer to Peer network of open educational system. Although OEN is in early stage of implementation, we can compare the network topology with the potential candidates. In our future works, the network topology may be changed based on practical requirements of the various implementations. Because of the heterogeneous nature of OEN (autonomous educational systems with different infrastructure and implementations are participants of the network), we deal with unstructured network and have limitation to enforce global pattern (to enforce each organisation to follow). Therefore there are a few choices for designing the network for OEN:

\section{Powerful Central Point}

One of the oldest solutions is to supply a centralised unit for supporting the network connections. This centralised design assumes a powerful central node that manages the whole network and keeps all information about all nodes. However, this solution has the classic weakness of a single point of failure, if the central node goes down the whole network collapses, as well as high cost for providing and maintaining the centralised unit. This increases as the number of nodes (participants) and associated network traffic increases.

\section{Unstructured (Fully) Distributed Network}

An unstructured Peer to Peer network is formed when the overlay links are established randomly.

In an unstructured Peer to Peer network, if a node wants to execute a transaction or even a simple search, the query has to be flooded through the network (will be sent to its neighbours and they will pass the request to their neighbours, etc.) to find as many nodes as possible that share the data. As a request may not get to all nodes in the network, the main disadvantage with such networks is that the request may fail to reach its desirable results (or find any result in term of search).

\section{Super Peers Networks}

The conventional solution to provide a stable Peer to Peer network, which has been used by several Peer to Peer networks (Yang \& Garcia-Molina, 2003), consists of introducing an extra layer to the network, the so-called super peers. Actually the super peers are de-centralised servers, which are intended to provide reasonable connectivity and avoid the fragmentation in the network. Depending on the size of the network, the protocol used, and the number of super peers, each super peer manages a number of nodes and can check their availability etc. At the same time, each super peer provides a strong link to the other super peers and in this way the design ensures that there is low probability for fragmentation.

\section{Our Scale-free Model}

Our solution (as been described in the previous section) relies on a network with the power-law distribution degree (which is called Scale-free network) when our link replication mechanism, increases the connectivity of each node when it executes a transaction. By increasing the connec- 
tivity of each node, we try to overcome one of the weaknesses of Scale-free network, which is weak connectivity on majority of nodes. This means if a node participates in more transactions (interacting more with other nodes), it will gain more links and will have better connectivity.

\section{Comparisons}

Providing centralised unit for OEN is not only expensive but also suffers a single point of failure (when the centralised unit is damaged or fails to respond, the connectivity between participants will be impossible). Furthermore there is a clear limitation on maximum nodes in the network, which depends on the centralised unit hardware and bandwidth.

Although unstructured Peer to Peer network seems interesting, there are two weaknesses: one that has been mentioned is related to processing a request and the other one is the amount of network traffic which will be produced during a query (especially if we deal with multi-media queries). Because the requester of a query has flooded the network, the data packages (query and its responses) will propagated in the network. This easily can cause a traffic bottleneck for the network.

In Super Peer Networks, super peers have to be stable nodes that can be online permanently and cope with huge amount of traffic. This means they are expensive nodes with costly maintenance requirements. It should also be noted that the resources are used for facilitating network operation management tasks. When considering such a solution for OEN, the question arises as to who is going to provide such nodes? Additionally, during peak time (when the interactions between participants are maximised) the pressure of high traffic can result in a bottleneck on super peer nodes and because of the connectivity role of super peers, the whole VSNs and consequently VPINs (and the possibility for collaboration of educational systems) will come under serious risk.

Our model, as Scale-free network, similar to Super Peer Networks, relies on a few nodes with majority of links (based on power-law distribution degree), but by applying link replication mechanism, other nodes will gain more links by involving in transactions with other nodes. This means in contrast with Super Peer Networks, we do not have fixed super peers. If a node interacts more with other nodes through link replication, it gathers more links and increase its connectivity. This means not only it may not need to rely on super peers (in scale-free network is called hubs) for its connectivity but during its life-time and by increasing its links, it may play the role of hub for other node. Furthermore our model as scale-free network can benefits small-world properties of a scale-free network (Barabási et al., 2000); the neighbourhood connectivity will increase (cluster coefficient will be close to 1) and the number of failed transactions will reduced.

\section{Conclusion}

In this paper, we have proposed an open education network, where institutions connect to each other, share their resources, and create new services. The proposed framework provides a conceptual design, where institutions can gather knowledge about other educational institutions' services, promote their own services, compose each other services, and regularly update their knowledge centres. Through our collaborative model, institutions can create new modules, courses, and qualifications.

While the currently prevalent classical method of educating students in centralised brick and mortar educational institutions is slowly giving way to the Internet-based online studies, progress (moving away from the centralized/traditional system to one of online system) is of course dependent on the availability of necessary infrastructure (ICT). The Internet has made this crossover possible and the virtual university using the internet can take the online studies a step further, decreasing the existing time-lag that exists between societal and technological changes that 
are taking place and accumulation and diffusion of the necessary knowledge through the existing educational institutions.

In contrast to the conventional electronic educational system that relies on the traditional datacentered knowledge approach, where the knowledge services can be accessed through a standard interface, such as a web page, we offer an interaction-centered approach to knowledge sharing that relies on the interaction models in a service-oriented architecture.

What we offer is a system where courses presented by one institution can be accessed and used (and even be included in a degree program offered) by one or several other institutions. In this way, any new or innovative course or program can be made instantly available to all who participate in sharing knowledge. It is hoped that our proposed system, while reducing the aforementioned time-lag, will also simplify access to the needed knowledge. In addition the OEN will allow for a much greater degree of customization of educational services that is currently possible, while reducing the overall cost of education for the public and businesses alike.

This research is of course a proposal and further work is needed. We have focused on the technical architectural vision in this paper. It would be highly risky to propose that such architecture be implemented in full without further evaluation. Our strategy is to incrementally build up experience, and then incrementally refine the architecture as necessary. Currently we are working with the Instituto de Pesquisas em Tecnologia e Inovação, Brazil, to implement a simplified architecture for to support high school education. In addition, while we continue to evaluate and refine the technical architecture, we also need to explore the accreditation and administrative issues with the presentation of educational resources from multiple institutions. The experience of one of us (Krause) with working with the BCS Information Systems Examinations Board (ISEB) is that a gradual internationalisation of accreditation processes, at least for Professional Development, is inevitable. This is already happening with the move to a European wide standard for software testing certification. This opens up the possibility for accreditation of courses by multiple providers against syllabi that are agreed on a European-wide basis.

\section{Acknowledgement}

This work has been part funded by the EPSRC supported Knowledge Transfer Account.

\section{References}

Addey, D., Suh, P., Ellis, J., \& Thiemecke, D. (2002). Content Management Systems. Peer Information. Retrieved from http://portal.acm.org/citation.cfm?id=863179

Barabási, A. L., \& Albert, R. (1999). Emergence of scaling in random networks. Science, $286(5439), 509$.

Barabási, A. L., Albert, R., \& Jeong, H. (2000). Scale-free characteristics of random networks: The topology of the world-wide web. Physica A: Statistical Mechanics and its Applications, 281(1-4), 69-77.

Bernstein, P. A., Hadzilacos, V., \& Goodman, N. (1987). Concurrency control and recovery in database systems. Boston, MA, USA: Addison-Wesley Longman Publishing.

Boley, H., \& Chang, E. (2007). Digital ecosystems: Principles and semantics. In Proceedings of the 2007 Inaugural IEEE Conference on Digital Ecosystems and Technologies (pp. 1-6).

Boyd, D. M., \& Ellison, N. B. (2007). Social network sites: Definition, history, and scholarship. Journal of Computer Mediated Communication (electronic edition), 13(1), 210.

Butler, B. S. (2001). Membership size, communication activity, and sustainability: A resource-based model of online social structures. Information Systems Research, 12(4), 346-362.

doi:10.1287/isre.12.4.346.9703 
Christensen, E., Curbera, F., Meredith, G., \& Weerawarana, S. (2001). Web services description language (WSDL) 1.1. W3C Web Site.

Curbera, F., Duftler, M., Khalaf, R., Nagy, W., Mukhi, N., \& Weerawarana, S. (2002). Unraveling the web services web: An introduction to SOAP, WSDL, and UDDI. IEEE Internet Computing, 6(2), 86-93.

Daho, Z. B., \& Simoni, N. (2006). Towards dynamic virtual private service networks: Design and selfmanagement. 10th IEEE/IFIP Network Operations and Management Symposium, 2006. NOMS 2006 (pp. 1-4).

Date, C. (2003). An introduction to data base systems (8th ed.). Pearson Education.

Elmagarmid, A. K. (1992). Database transaction models for advanced applications. San Francisco, CA, USA: Morgan Kaufmann.

Gray, J. (1992). Benchmark handbook: For database and transaction processing systems. San Francisco, CA, USA: Morgan Kaufmann.

Gray, J., \& Reuter, A. (1993). Transaction processing: Concepts and techniques. San Francisco, CA, USA: Morgan Kaufmann.

Haghjoo, M. S., Papazoglou, M. P., \& Schmidt, H. W. (1993). A semantic-based nested transaction model for intelligent and cooperative information systems. Proceedings of International Conference on Intelligent and Cooperative Information Systems, 321-331.

Kakeshita, T., \& Xu, H. (1992). Transaction sequencing problems for maximal parallelism. Second International Workshop on Research Issues on Data Engineering, 1992: Transaction and Query Processing, 215-216.

Kelsey, T. (2010). Social networking spaces: From Facebook to Twitter and everything in between. USA: Apress.

Krause, P., Marinos, A., Moschoyiannis, S., Razavi, A., Zheng, Y., Kurtz, T., Streeter, M. P., \& Gabaldón, J. E. (2008). Full architecture definition for autopoietic P2P network version 1. Project acronym: OPAALS, European Community, Framework 6, Contract No: 034824. Retrieved from http://files.opaals.org/OPAALS/Year 2 Deliverables/WP03/D3.3.pdf

Kristiansen, L., Hansen, P. F., \& Licciardi, C. (2008). TINA 1.0 deliverables and specifications. Retrieved March 25, 2009, from http://www.tinac.com/specifications/specifications.htm

Li, Z., \& Mohapatra, P. (2004). QRON: QoS-aware routing in overlay networks. IEEE Journal on Selected Areas in Communications, 22(1), 29-40.

Light, V., Light, P., \& Wright, V. (2000). Seeing eye to eye: An evaluation of the use of videoconferencing to support collaboration. European Journal of Psychology of Education, 15(4), 467-478. doi:10.1007/BF03172988

Martin, D., Burstein, M., Hobbs, J., Lassila, O., McDermott, D., McIlraith, S., . . Sycara, K.. (2004). OWL-S: Semantic markup for web services. Retrieved from http://www.ai.sri.com/daml/services/owls/1.1/overview

Martini, B., Baroncelli, F., \& Castoldi, P. (2005). A novel service oriented framework for automatically switched transport network. IM 2005. 2005 9th IFIP/IEEE International Symposium on Integrated Network Management, 295-308.

Moss, J. E. B. (1985). Nested transactions. Cambridge, Mass: MIT Press.

Nachira, F. (2002). Towards a network of digital business ecosystems fostering the local development. European Commission discussion paper. Bruxelles.

Papazoglou, M. P., \& Georgakopoulos, D. (2003). Service-oriented computing. Communications of the $A C M, 46(10), 25-28$.

Papazoglou, M. P., Traverso, P., Dustdar, S., Leymann, F., \& Kramer, B. J. (2006). Service-oriented computing: A research roadmap. Service Oriented Computing (SOC). 
Ramakrishnan, R., \& Gehrke, J. (2003). Database management systems. McGraw-Hill Science/Engineering/Math.

Razavi, A. R. (2009). DIGITAL ECOSYSTEMS: A distributed service oriented approach for business transactions. (PhD Thesis, University of Surrey).

Razavi, A. R., Moschoyiannis, S., \& Krause, P. (2007a). A coordination model for distributed transactions in digital business ecosystems. Digital Ecosystems and Technologies (DEST 2007), IEEE Computer Society Press, Los Alamitos.

Razavi, A. R., Moschoyiannis, S., \& Krause, P. (2007b). Preliminary architecture for autopoietic P2P network focusing on hierarchical super-peers, birth and growth models. OPAALS Project (OPAALS project Deliverable D3.1). Retrieved from http://files.opaals.org/OPAALS/Year 1 Deliverables/WP03/OPAALS D3.1-final_submitted.pdf

Razavi, A. R., Moschoyiannis, S., \& Krause, P. (2007c). Concurrency control and recovery management for open e-business transactions. Communicating Process Architectures 2007: WoTUG-30: Proceedings of the 30th WoTUG Technical Meeting, 8-11 July 2007, University of Surrey, Guildford, United Kingdom (p. 267). Ios Pr Inc.

Razavi, A. R., Moschoyiannis, S., \& Krause, P. (2008). A scale-free business network for digital ecosystems. Proceedings IEEE Digital Ecosystems and Technologies (IEEE-DEST 2008).

Siebes, R., Dupplaw, D., Kotoulas, S., Perreau de Pinninck, A., van Harmelen, F., \& Robertson, D. (2007). The OpenKnowledge System: An interaction-centered approach to knowledge sharing. On the Move to Meaningful Internet Systems 2007: CoopIS, DOA, ODBASE, GADA, and IS, 381-390. Retrieved from http://dx.doi.org/10.1007/978-3-540-76848-7_25

Singh, M. P., \& Huhns, M. N. (2005). Service-oriented computing: Semantics, processes, agents. Wiley.

Sivashanmugam, K., Verma, K., Sheth, A., \& Miller, J. (2003). Adding semantics to web services standards. Proceedings of the International Conference on Web Services, 395-401.

Sycara, K., Paolucci, M., Ankolekar, A., \& Srinivasan, N. (2003). Automated discovery, interaction and composition of semantic web services. Web Semantics: Science, Services and Agents on the World Wide Web, 1(1), 27-46.

Weedman, J. (1999). Conversation and community: The potential of electronic conferences for creating intellectual proximity in distributed learning environments. Journal of the American Society for Information Science, 50(10), 907-928. doi:10.1002/(SICI)1097-4571(1999)50:10<907::AIDASI7>3.0.CO;2-R

Wellman, B., Salaff, J., Dimitrova, D., Garton, L., Gulia, M., \& Haythornthwaite, C. (1996). Computer networks as social networks: Collaborative work, telework, and virtual community. Annual Review of Sociology, 22(1), 213-238.

Yang, B., \& Garcia-Molina, H. (2003). Designing a super-peer network. Proceedings of the International Conference on Data Engineering (pp. 49-62). IEEE Computer Society Press; 1998. 


\section{Biographies}

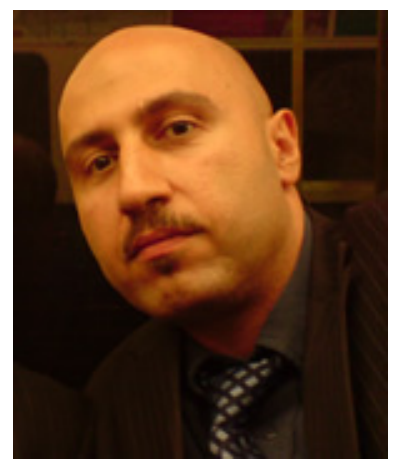

Amir reza Razavi is a research associate at the University of Surrey, where he leads the Virtual University project. He has a BSc in Software Engineering (Shiraz Azad University 1996-Iran), two MScs (Iran University of Science and Technology 1999-Iran \& University of Surrey 2003-UK) and a $\mathrm{PhD}$ on computer science (University of Surrey 2009-UK). His main interest has been distributed computing, longrunning transactions in non-conventional environments and interaction models for educational systems. He has contributed in several EU projects (OPAALS, DBE and ASPIC) and currently designs an open source educational framework for Virtual University project.

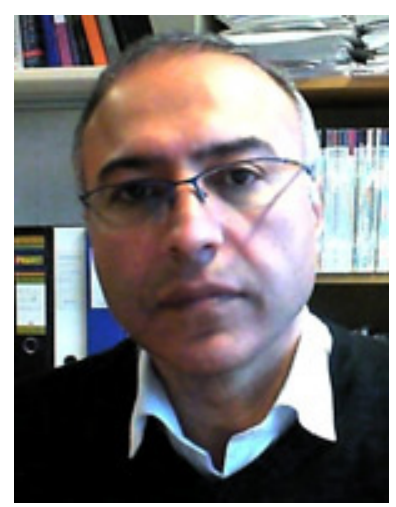

Abbas Strømmen-Bakhtiar is an associate professor at the Centre for Enterprise Architecture and Information Systems, Graduate School of Business, University of Nordland. He has BScs in Aerospace Engineering Technology and Computer Science, MScs in Information Technology and Computer Integrated Manufacturing and a PhD in Strategic management. His main interests are in the areas of Management Information Systems and Strategic Management. He is currently working on several projects such as open source educational framework for Virtual University, the application and use of decision support systems for managing operations in extreme environments.

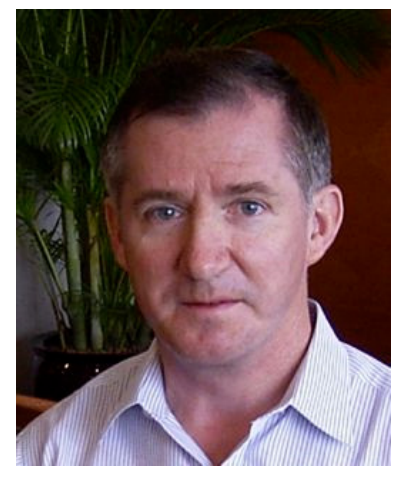

Paul Krause has spent a lifetime studying complex systems in physics, mathematics, and computer science. In recent years he has been integrating his 25 years of experience as a volunteer in the environmental and rural sustainability arenas with his professional research. A specific example of this is his involvement with the 6-year project, Evolution and Resilience in Industrial Ecosystems (ERIE). ERIE is funded under the Research Council UK Complexity in the Real World programme. Paul leads the policy formulation research stream in ERIE with two case studies under way: one exploring evolution and transformation in the South Humber Bank region; and, one in food security. In the latter we take a strong stance on sustainability of food supply as requiring full accounting of all five capitals (Social, Natural, Human, Physical, as well as Financial) of the Sustainable Livelihoods Approach (SLA). 